\title{
The effect of geometry and illumination on appearance perception of different material categories
}

\author{
Bin Chen ${ }^{1}(\mathbb{D}) \cdot$ Chao Wang $^{1} \cdot$ Michal Piovarči ${ }^{2} \cdot$ Hans-Peter Seidel ${ }^{1} \cdot$ Piotr Didyk $^{3} \cdot$ Karol Myszkowski $^{1}$. \\ Ana Serrano ${ }^{1,4}$
}

Accepted: 20 June 2021 / Published online: 14 July 2021

(c) The Author(s) 2021

\begin{abstract}
The understanding of material appearance perception is a complex problem due to interactions between material reflectance, surface geometry, and illumination. Recently, Serrano et al. collected the largest dataset to date with subjective ratings of material appearance attributes, including glossiness, metallicness, sharpness and contrast of reflections. In this work, we make use of their dataset to investigate for the first time the impact of the interactions between illumination, geometry, and eight different material categories in perceived appearance attributes. After an initial analysis, we select for further analysis the four material categories that cover the largest range for all perceptual attributes: fabric, plastic, ceramic, and metal. Using a cumulative link mixed model (CLMM) for robust regression, we discover interactions between these material categories and four representative illuminations and object geometries. We believe that our findings contribute to expanding the knowledge on material appearance perception and can be useful for many applications, such as scene design, where any particular material in a given shape can be aligned with dominant classes of illumination, so that a desired strength of appearance attributes can be achieved.
\end{abstract}

Keywords Perception - Material appearance

\section{Introduction}

Bin Chen

binchen@mpi-inf.mpg.de

Chao Wang

chaowang@mpi-inf.mpg.de

Michal Piovarči

michael.piovarci@ist.ac.at

Hans-Peter Seidel

hpseidel@mpi-sb.mpg.de

Piotr Didyk

piotr.didyk@usi.ch

Karol Myszkowski

karol@mpi-inf.mpg.de

Ana Serrano

anase@unizar.es

1 Max-Planck-Institut für Informatik, Saarbrücken, Germany

2 Institute of Science and Technology Austria, Klosterneuburg, Austria

3 Universitả della Svizzera Italiana, Lugano, Switzerland

4 Centro Universitario de la Defensa, Zaragoza, Spain
The perception of material appearance is a long-standing psychovisual problem, which is not yet fully understood $[2,9]$. One of the main reasons is the complex interaction and entanglement between material reflectance, geometry surface, and illumination. The appearance of a given material may change drastically for different shapes or under different illuminations $[23,45]$. The unlimited combinations between these three factors create multitudinous material appearances that make the study of this problem harder.

Many works have approached this problem [2,10,13,28]. Most of them focus on investigating some particular aspects of material appearance, such as gloss [5,35] or translucency $[11,14]$. The effects of geometry and illumination have also been studied, but usually on simplified geometries under a limited variety of illuminations $[1,26,32,34,36,43]$. Finally, there are some recent works devoted to exploring a wider variety of appearances; however, they do not take into account the impact of geometry and illumination [17,40]. In order to be able to investigate the interaction of material, shape, and illumination for a wide variety of appearances 
and derive more general insights, the recent work of Serrano et al. [39] presented a diverse collection of 42,120 images with distinct combinations of measured BRDFs, shapes, and real-world illuminations with their corresponding perceptual ratings for six appearance attributes: lightness, metallicness, anisotropy, as well as gloss-related: glossiness, sharpness of reflections and contrast of reflections.

In this work, we make use of this dataset to provide, for the first time, an in-depth analysis of the impact of the interactions between material category, illumination, and geometry in perceived appearance attributes. To this end, we label a set of materials with eight representative material categories. Then, we perform two statistical analyses using CLMM for robust regression. First, we analyze the material category, geometry and illumination as independent fixed factors. Then, based on this analysis, we select a representative subset of the data to investigate the complex interactions between material category, geometry, and illumination. We observe a number of interesting interactions, e.g., while the metallicness attribute is consistently stronger for metals in all tested conditions, the plastics and ceramics categories may achieve comparable strength of the gloss-related attributes under low-frequency illuminations and smooth, low curvature geometries. Conversely, strong directional illumination seems to be most effective in increasing appearance differences between the material categories.

We believe that our findings contribute to further understanding the processes that drive material perception and that our insights can be useful as guidelines for design applications where material category, geometry, and illumination interact with each other.

\section{Related work}

In this work, we explore the effect of geometry and illumination on the perception of material appearance for different material categories. Lightness (albedo), glossiness, and metallicness have been consistently identified as the key attributes of material perception [10,42]. In general, the human visual system (HVS) aims toward material perception constancy, which often holds for lightness [34]. Therefore, in this work we focus on the perception of glossiness [5] and metallicness [41] that show high variability across material category, illumination, and geometry.

Glossiness Perceived glossiness strength increases with brightness and contrast of highlights, their relative coverage on the surface, and the sharpness of the reflected environment $[26,28]$. These factors directly depend on material reflectance properties, e.g., metals tend to reflect strongly only near specular directions, while dielectrics typically involve some Lambertian reflections that reduce highlight contrast and perceived reflection sharpness [41]. Illumina- tion plays an important role as well, e.g., strong directional lights increase contrast of highlights, while distinctive environment patterns improve sharpness of reflections, so that overall perceived glossiness increases as well $[1,6,31,36,45]$. Conversely, uniform ambient illumination typically reduces perceived glossiness, but in some scene configurations it might contribute to an increase of the highlight coverage and this way enhance perceived glossiness [26,41]. The judgment of glossiness becomes the most intuitive when objects are illuminated by captured high-contrast high-dynamic range (HDR) environment maps [1,12], while oversimplified synthetic lighting might be more confusing in understanding material properties.

Although the effect of geometry has been less explored than the effect of illumination, evidence suggests that perceived glossiness of surfaces in natural lighting environments depends on the structure of specular contours [22]. Results presented by Marlow et al. [29] demonstrate that the HVS exploits surface curvature to compute the relative scatter of a surface's reflectance function. In the real world, increasing surface curvature leads to spatial compression of highlights, so that their intensity increases [21]. In displayed images, this effect might not be directly reproduced due to limited display luminance, or clamping highlight details due to tone mapping. In the latter case, the highlight coverage might increase and this way enhance perceived glossiness [26,41]. Bumpy surfaces also might increase such highlight coverage, but when their structure is too fine, perceived sharpness of reflections and their contrast might be reduced, so that the overall perceived glossiness might be reduced as well $[24,28]$. In the context of material discrimination, Vangorp et al. [43] showed that curvature captures highlights better than tessellated shapes and therefore allows for better material discrimination.

Metallicness As metallicness is also highly directional attribute, many observations presented here for glossiness hold as well. Metallicness might be stronger for ambient illumination from a wide range of directions (which is the case for environment map lighting), but it also increases with a larger highlight coverage, higher highlight sharpness and contrast [26,41]. Specular reflections for metals are much stronger than for plastic and they may cover larger surface regions, while practically there are no diffuse reflections.

All discussed here works considered selected glossiness and metallicness appearance factors using a few distinct materials, typically represented by analytic reflectance models. Simultaneous interactions between multiple factors have been relatively sparsely investigated. A notable exception is work of Serrano et al. [39], where a large-scale crowdsourced Web experiment has been performed using measured BRDFs to derive human ratings for the appearance attributes. While the key goal of this work was to train a neural network to predict such attributes, also statistical analysis has been 


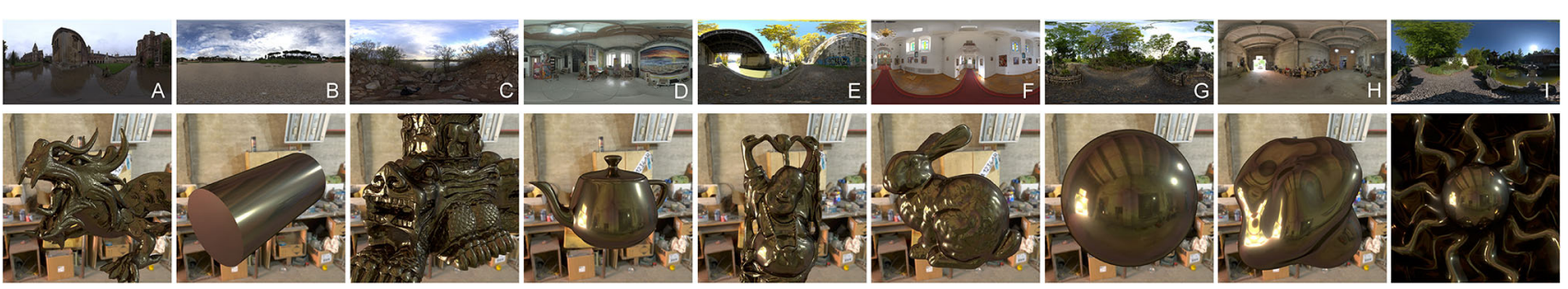

Fig. 1 Examples of the nine illuminations and geometries in the dataset collected by Serrano et al. [39]. In the bottom row, the brass BRDF from MERL dataset has been used for rendering

performed to investigate whether illumination and geometry have a significant impact on human's ratings. In this work, we employ the same rating data, but for the first time we investigate interactions of illumination and geometry with different material categories.

Perceptual gloss embeddings An important practical problem is to identify meaningful perceptual dimensions that are suitable for gloss appearance editing. Pellacini et al. [35] and Wills et al. [44] employed dimensionality reduction techniques (such as multi-dimensional scaling) in analytic and measured BRDFs [30]. This research suggests that two perceptual gloss dimensions: (1) distinctness-of-image gloss, i.e., sharpness of the image reflected in surfaces, and (2) contrast gloss, i.e., contrast between specularly reflecting areas and other areas, as originally introduced by Hunter $[18,19]$, allow to control the gloss appearance. In this work, we consider those gloss dimensions and following Serrano et al. [39] we refer to them as sharpness of reflection and contrast of reflection. Recently, data-driven methods have been employed to derive intuitive appearance editing spaces [40] and deep BRDF representations [17].

\section{Experiment}

The goal of this paper is to investigate the effect of illumination and geometry in perceived appearance for different material categories. For completeness, in this section we briefly summarize the details of the experiment carried out by Serrano et al [39] for collecting crowdsourced data, since we use their dataset for performing our analysis. Then, we motivate our selection of a subset of this dataset for performing an in-depth analysis of perceived attributes for different material categories under different illuminations and geometries.

\subsection{Dataset summary}

We make use of the dataset collected by Serrano et al. [39], which contains 42,120 images with combinations of 520 measured BRDFs, 9 geometries and 9 illuminations. Figure 1 shows all HDR environment maps and geometries in the dataset. This dataset contains crowdsourced subjective ratings collected with Amazon Mechanical Turk with a 7-point Likert rating task [33,40]. A total of 3, 217 participants (37\% female, average age 38.1, $\sigma=11.96$ years old) participated in the study, and as a result, 215,680 valid responses were collected, with at least 5 ratings for each different image. This is the largest dataset to date containing comprehensive information about perceived attributes for different geometries, illuminations, and materials.

Attributes Originally, the following perceptual attributes have been considered for each image: metallicness, glossiness, contrast of reflections, sharpness of reflections, lightness, and anisotropy. As mentioned in Sect. 2, we are less interested in lightness that shows a good constancy across different illuminations and geometries. Also, we ignore anisotropy due to relatively sparse representation in the dataset as acknowledged in [39]. Effectively, in this work we consider the following four attributes: metallicness, glossiness, contrast of reflections, and sharpness of reflections. Please refer to Sect. 2 for more details on those attributes and their importance in material perception.

\subsection{Labeling material categories}

Materials Following the availability of perceptual ratings from [39], we consider a number of material BRDF databases as detailed in Table 1. As the focus of this work is the effect of geometry and illumination for different material categories, we label materials in those databases and finally select eight representative categories: wood, leather, fabric, paper, natural, plastic, ceramic, and metal. This particular choice is motivated by rich representation of such materials in those databases. We decide to ignore possibly interesting categories [38] with too sparse representation. As a result, we select a subset of 363 materials (183,352 trials) for our analysis. We trained our first CLMM model on all the 8 material categories, 9 geometries, and 9 illuminations and analyze it in Sects. 4.1 and 4.2. 
Table 1 Material category labelling details

\begin{tabular}{llllllllll}
\hline & Wood & Leather & Fabric & Paper & Natural & Plastic & Ceramic & Metal & Others \\
\hline MERL [30] & 0 & 0 & 14 & 5 & 12 & 29 & 6 & 26 & 8 \\
MERL-EDIT [39] & 0 & 0 & 16 & 0 & 28 & 0 & 0 & 19 & 125 \\
RGL [7] & 0 & 0 & 8 & 10 & 2 & 1 & 0 & 19 & 22 \\
UTIA [8] & 14 & 12 & 85 & 3 & 0 & 5 & 0 & 0 & 1 \\
UTIA-ISO [8,39] & 1 & 4 & 41 & 2 & 1 & 0 & 0 & 0 & 1 \\
\hline
\end{tabular}
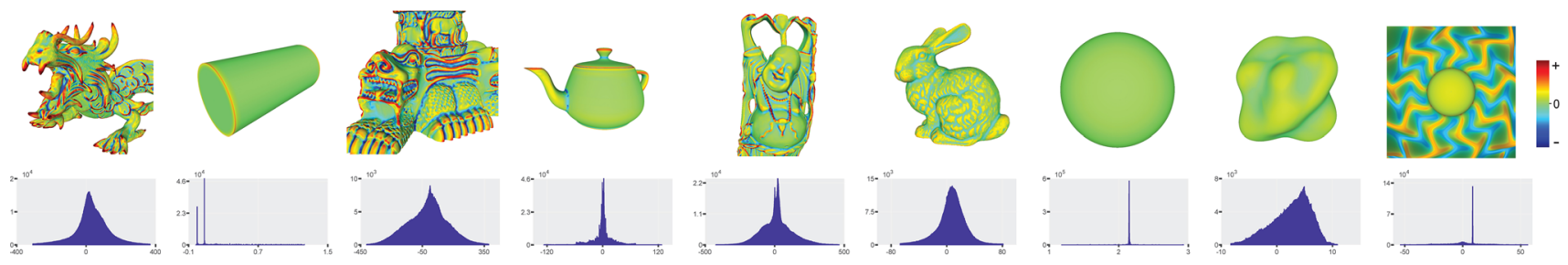

Fig. 2 The mean curvature on all 9 geometries calculated by the algorithm proposed by [15] with MLS filter scale 20. Red and blue represent the convex and concave surfaces, respectively

\subsection{Selecting subsets of geometry and illumination}

Geometry The surface curvature is an important indicator that reveals the influence of geometry on material perception [3,20,25-27]. Specifically, from the rendering perspective, the local surface curvature is related to the local distribution of normal vectors, which further influences the interaction between viewing point, illumination, and material properties, and finally determine the perceptual appearance. Marlow et al. [26] applied the grand mean of curvature to represent the complexity of a geometry and suggested the increase in local surface curvature would enhance the specular sharpness but depress the specular coverage. In this paper, we focus on the histogram of local curvature and several statistical characteristics to analyze the effect of geometry on material appearance perception. Figure 2 shows the 9 geometries in the dataset colorized by the values of local mean curvature calculated using the algebraic point set surface (APSS) algorithm [15] with moving least-square (MLS) filter scale equal to 20. The corresponding statistical histogram can also be found below each geometry. Note that the geometries have been normalized into same scale, and curvature values calculated here have been adjusted based on the relative density of vertices (where dragon has the largest amount of vertices so with density equal to 1 as reference) so all the geometries are comparable.

Among all 9 geometries, dragon has the most complex surfaces and overall lowest rating; buddha contains the most diverse geometry components; blob has a gently changing smooth surface and is considered as one of the best choices for material discrimination [43]; the sphere component and wavy region make ghost the best for single image material comparison [16]. Thus, we select these four geometries for further analyzing the interaction between material category, geometry and illumination in our second CLMM model.

Illumination Among the 9 environment maps in the dataset, $I$ has a strong directional light with the highest dynamic range with respect to the rest of environment maps; $E$ contains distinct area light sources; $G$ includes a large area light and lots of small point-like light sources; $A$ features relatively large regions of slowly changing illumination, e.g., around the sky. In general, $A, E, G$, and $I$ cover a large range of high-frequency content (from smallest in $A$ to largest in $I$ ). Therefore, we select these four environment maps for our second CLMM model with interaction.

\section{Data analysis}

In this section, we perform our analysis and describe our findings. Since the dependent variables (attributes) are 7point Likert items and the collected observations are not normally distributed ( $p<0.05$ for the Kolmogorov-Smirnov test), we use CLMM to analyze ordinal-scale observations without treating these variables as quantitative. The Estimated Marginal Means (EMM) with Bonferroni correction are selected for the post hoc analysis. The illumination, geometry, and material category are treated as fixed factors, and the user and material as mixed effects for our CLMM model. We use this model for analyzing the effect of material category, geometry, and illumination in Sects. 4.1 and 4.2 , respectively.

Then, we compute another CLMM model for analyzing the complex interactions of material category, geometry, and illumination. We use a subset of the data to allow the model to converge, since using all possible pairwise interactions between these three factors would become intractable. After 


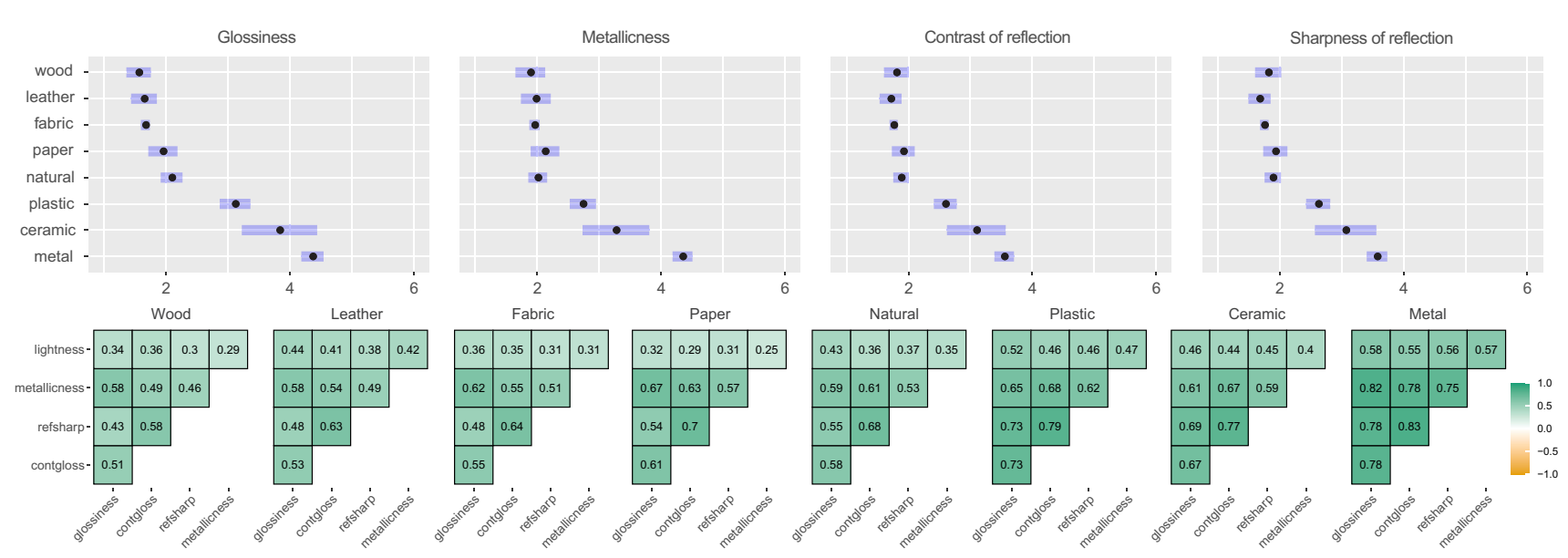

Fig. 3 Top row: the Estimated Marginal Means values of material category for five material appearance attributes, where black points represent the mean value, and the blue bars indicate a 95\% confidence interval. Bottom row: Spearman rank correlations for the appearance attributes in each material category

our analysis in Sect. 3.3, we use a subset of four illuminations, in particular $A, E, G$, and $I$, as well as four geometries: dragon, buddha, blob, and ghost. For materials, we focus on the MERL dataset [30] since it has the most diverse material categories. After our initial analysis in Sect. 4.1, we select four material categories: fabric, plastic, ceramic, and metal that cover the largest range of all perceptual attributes. We analyze this interaction model in Sect. 4.3.

\subsection{Effect of material category}

There is a significant effect of the material category on the perceptual material attributes $(p<0.001)$. We show the post hoc Estimated Marginal Mean (EMM) effects of material categories on our four attributes in the first row of Fig. 3. Note that the EMM effects show relative changes with respect to the average model: they account for the estimated effects of material category on isolation, across all variety of geometries and illuminations. There are no significant differences observed between wood, leather, fabric, paper, and natural materials. Those five material categories can be treated as one group, which together with plastic, ceramic, and metal presenting a monotonic trend of increasing attribute rating. The large confidence interval for ceramics is due to a small number of such materials in our dataset (Table 1).

We also analyze Spearman rank correlations between the users' ratings for the perceptual attributes on each material category, shown in the bottom row of Fig. 3. Our perceptual data do not show obvious correlations for wood, leather, fabric, paper, and natural materials. As expected, for plastic, ceramic, and metal, the gloss-related attributes (glossiness, contrast of reflection and sharpness of reflection) show high correlations. Based on the outcome of EMM and correlation analysis, fabric has been selected as a material category that represents well wood, leather, paper, and natural materials with respect to the gloss-related attributes.

Additionally, we compute the average BRDFs for fabrics, plastics, ceramics, and metals to get some insights about the basic characteristics of each material category. In Fig. 5-right we show the profiles of averaged BRDFs in polar coordinates. We parameterize the averaged BRDFs to the half-angle $\theta_{h}$ representation proposed by Rusinkiewicz [37] to get a better insight about the reflectance transition from the specular peak to grazing angles. A slice with the difference angles $\theta_{d}=45$ and $\phi_{d}=90$ [4] for each averaged BRDF is shown in Fig. 5-left. Metal has the highest specular peak when $\theta_{h}=0$, but the reflectance falls down stronger than for other material categories with increasing $\theta_{h}$. Plastic and ceramic show similar trends except the grazing angles $\left(\theta_{h}\right.$ near $\left.90^{\circ}\right)$, where Fresnel effects can be observed. Fabric shows the lowest specular peak but highest Fresnel effect near grazing angle. Figure 4 demonstrates the rendered images using our four averaged BRDFs on two extreme geometries (dragon and ghost) under two extreme illuminations ( $A$ and $I$ ).

We also explore further the relationships between each perceptual attribute on the collected data for different material categories. Thus, we average the subjective ratings on each stimuli and create 2D embeddings of perceptual attributes pairs for different material categories, shown in Fig. 6. An interesting nonlinearity can be observed on some combinations: glossiness-sharpness of reflections and glossiness-contrast of reflections for plastic, which is also the case for ceramic and metal (refer to the supplementary for all embedding combinations). This suggests that the sharpness of reflections and contrast of reflections start to be observed when glossiness is high enough for specular materials such as plastic, ceramic, and metal [39]. The embedding of sharpness of reflections and contrast of reflection on metals 


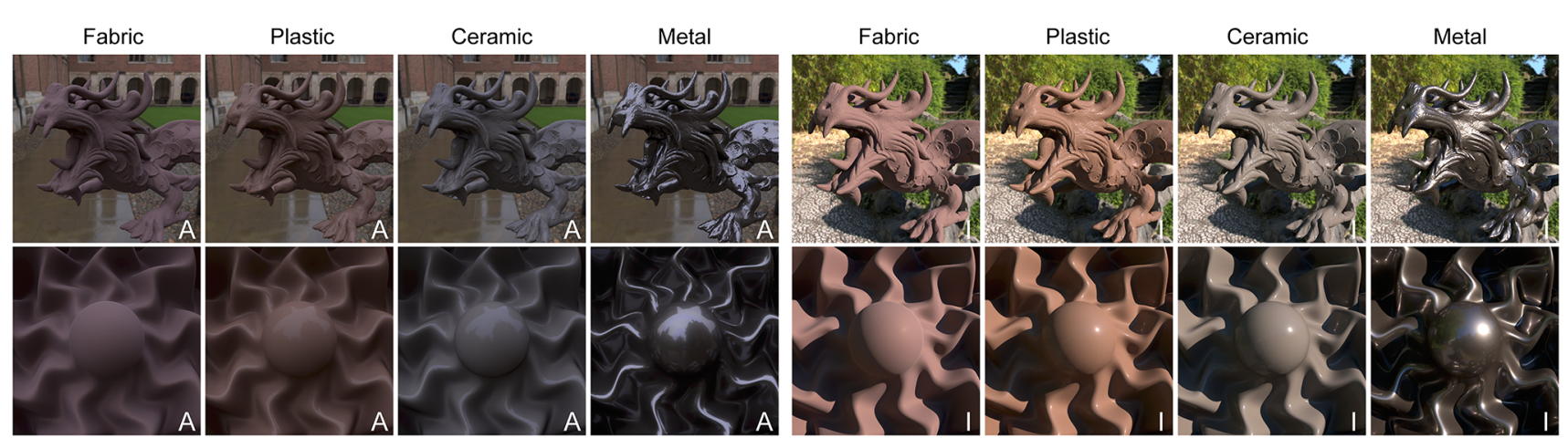

Fig. 4 Rendered images using our four averaged BRDFs (refer to Fig. 5) for dragon and ghost geometries under illuminations $A$ and $I$

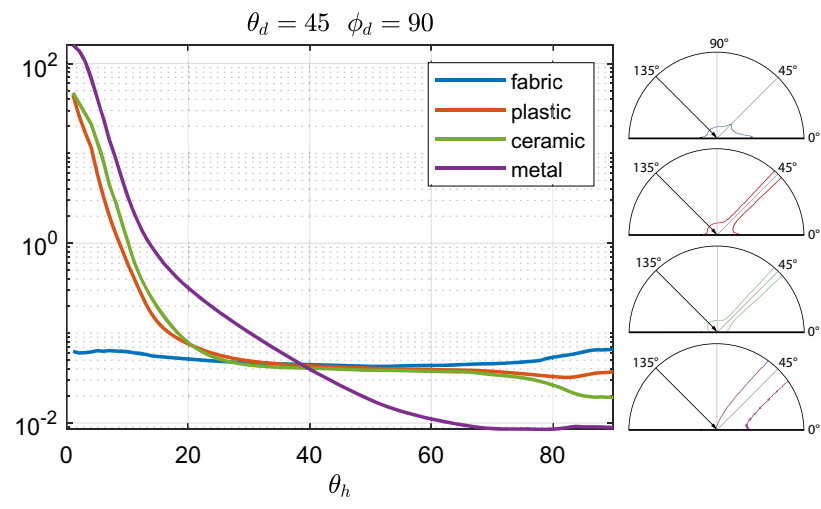

Fig. 5 Averaged BRDFs of four material categories. A BRDF slice for the fixed difference angles $\theta_{d}=45$ and $\phi_{d}=90$ is shown (left), and the corresponding polar plots (right)

(Fig. 6-right) indicates that sharpness becomes more visible only for larger contrast of reflections. Such larger contrast is characteristic for more specular material, where the surround of typically clamped highlights is darker and makes the reflected environment better visible [41]. There is no obvious relationship among the gloss-related attributes on fabric. Our embedding reveals the relationships among perceptual attributes that can be useful for perceptual material editing and design. Please refer to the supplementary for more results.

\subsection{Effect of geometry and illumination}

Geometry In order to derive insights about the observed effects of geometry, we compute a set of geometry features for describing our selected geometries. Based on the curvature distributions computed in Sect. 3.3, we compute the standard deviation $\left(G_{\text {std }}\right)$, kurtosis $\left(G_{\text {kurt }}\right)$, and median $\left(G_{\text {median }}\right)$ to characterize our four geometries. We choose median over mean to improve robustness toward outliers. In Fig. 7-right, we show the impact of these features in the estimated marginal effects for each of our four attributes. All the four perceptual attributes analyzed monotonically increase as the median curvature $\left(G_{\text {median }}\right)$ decreases. The decreasing median curvature indicates an overall smooth surface that will enlarge the coverage of specular highlight and further enhance the perceived glossiness and other gloss-related attributes [26]. Although the $G_{\text {std }}$ and $G_{\text {kurt }}$ do not show so determinate correlation to the perceived appearance as $G_{\text {median }}$, they follow a similar trend.

Illumination Similarly, we compute a set of illumination features and relate them to the observed effects in perceptual attributes. As we use HDR environment maps for illumination during rendering, we directly calculate the following statistics on four environment maps ( $A, E, G$, and $I$ ): standard deviation $\left(I_{\text {std }}\right)$, skewness $\left(I_{\text {skew }}\right)$, kurtosis $\left(I_{\text {kurt }}\right)$ light source coverage $\left(I_{\text {area }}\right.$, where small values correspond to point-like light sources), contrast between light sources and the rest of image ( $\left.I_{\text {range }}\right)$, and high-frequency content $\left(I_{\mathrm{hfc}}\right)$. Results are shown in Fig. 7-left. Our data confirm literature findings that gloss perception becomes stronger for more directional and higher intensity light sources (low $I_{\text {area }}$ and high $I_{\mathrm{hfc}}$ ) $[6,36,45]$. The perceived glossiness is also consistent with increasing environment map contrast (high $I_{\text {std }}$ and $I_{\text {range }}$ ) as also reported in $[1,31]$. A positive correlation between the skew of environment map histogram and perceived gloss has been reported by Adams et al. [1]. The skew and other statistical moments in Fig. 7-left also correlate well with perceived gloss. However, the environment map $(G)$ is an outlier here as its statistical moments are consistently higher than for $(E)$, while the perceived gloss is similar. This different behavior can be better explained by variations in $I_{\text {area }}$ and $I_{\mathrm{hfc}}$.

\subsection{Interaction between illumination, geometry, and material category}

In this section, we compute and analyze a second CLMM model with the interaction between material category, illumination, and geometry. 
Fig. 6 Stimuli embeddings for different perceptual attribute pairs for the plastic (left and center) and metal (right) categories
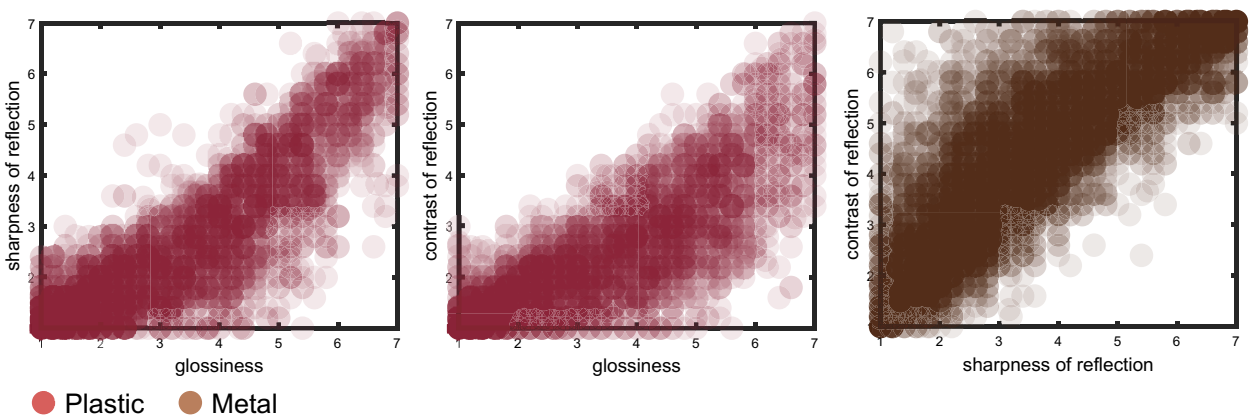

Plastic Metal
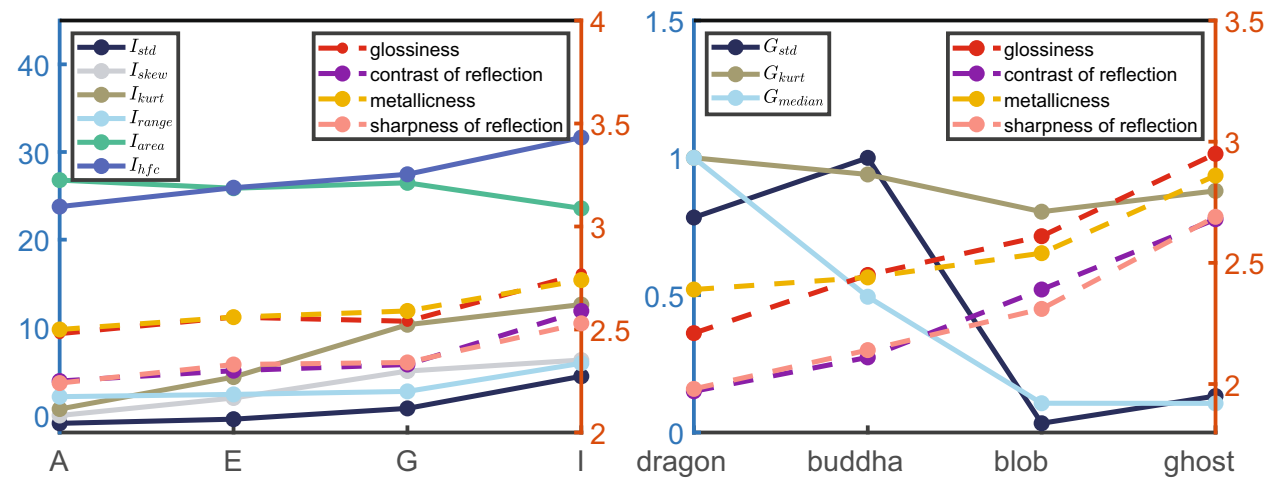

Fig. 7 Illumination and geometry features and their impact on the estimated marginal effects for glossiness, metallicness, sharpness of reflections, and contrast of reflections attributes. The estimated marginal effects approximate the response for each attribute, for each geometry and illumination in isolation (adjusted by other variables in the model)

\subsubsection{Glossiness}

Perceived gloss increases with the highlight coverage, sharpness, contrast, and brightness that in complex ways depend on illumination spatial structure, material properties, and surface geometry [26]. For example, the highlight coverage can be extended by blurring environment maps, expanding lobes of glossy reflection (material roughness), reducing surface curvature, making the viewpoint position perpendicular to the surface, to name just a few possible manipulations to achieve this goal. Here, we consider gloss variations due to all those factors for metals, ceramics, plastics, and fabrics.

As seen in Fig. 8-a, the gloss perception is strongest for metals, but some inconsistencies can be clearly observed. For example, the blob illuminated by $A$ and $E$ environment maps might be perceived even comparably glossy to ceramics and plastics. In contrast to more complex geometries such as the dragon and buddha, the blob features larger smooth surface regions (smaller $G_{\text {median }}$ in Fig. 7-right) that enable a more intuitive judgment of reflection sharpness (Fig. 12) and contrast (Fig. 10). This can be observed in Fig. 11. Note that for slowly changing illumination $A$ and $E$ (smaller $I_{\text {std }}$ and $I_{\text {range }}$ in Fig. 7-left) such reflections appear blurry, of low contrast and similar coverage, regardless whether this is metal, ceramic, or plastic material. Note that for ghost, the highlight coverage for metal is enlarged with respect to the ceramic and plastic, in particular, due to the wavy regions surrounding the central hemi-sphere that somehow enhances perceived glossiness. Overall slowly changing illumination (as $A$ and $E$ ) in interaction with slowly changing, smooth surfaces (as the blob) might reduce the differences in glossiness perception between metals and plastics/ceramics. On the other hand, the absolute magnitude of perceived glossiness is the highest for blob and ghost, mostly, regardless of illumination (refer also to Fig. 8-b).

We show results of glossiness pairwise comparison on interaction between material category and geometry in Fig. 8-c. Four material categories show similar trend as in Fig. 3 under the same geometry. When observing across different geometries, the value differences between fabric and other materials show negative correlation with the complexity of geometry. It seems the tesselated-like surfaces on dragon reduce the perceived glossiness on all materials, especially on dielectric specular materials such as plastic and ceramic. This is consistent with the finding of Vangorp et al. [43]. It seems the geometry complexity has less influence on metal compared to other materials.

\subsubsection{Metallicness}

As expected, the metallicness attribute is consistently strongest for metal over other material categories regardless of geometry and illumination (Fig. 9). The large gap between metal and other material categories lies in the reflectance differences between metal and dielectrics as seen in Fig. 5. Despite display brightness limitations and contrast-compressive tone mapping, the intensity of specular reflection is consistently the highest for metals (refer also to Figs. 4 and 11) due to 
Fig. 8 Estimated Marginal Means effects of glossiness for the interaction between $\mathbf{a}$. geometry, illumination, and material category (the height of each colored bar represents the value); b. material category and illumination; and c. material category and geometry

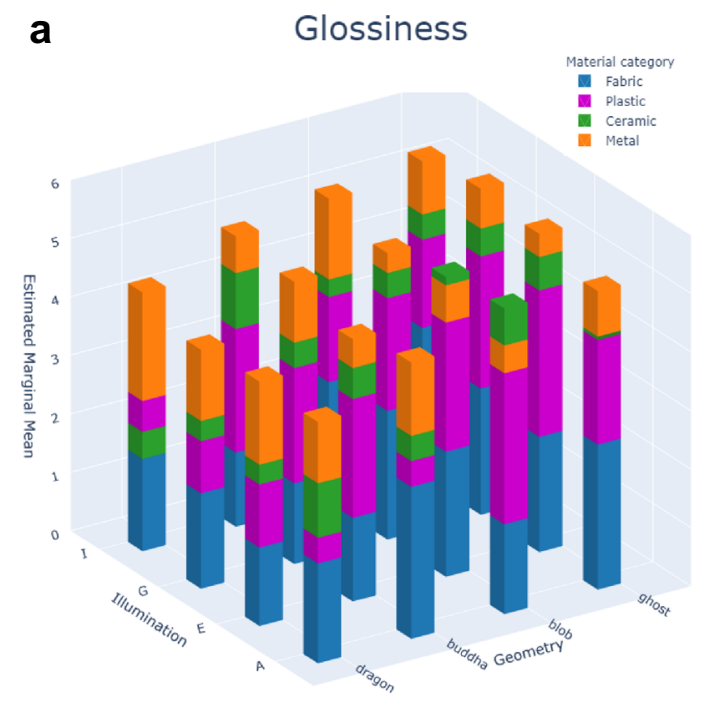

largest magnitude of BRDF for specular angles (small $\theta_{h}$ ), which is one of the key factors that enable to distinguish metals from plastic and other materials [41].

It seems the illumination has limited influence on metallicness when observing across different environment maps on all materials (Fig. 9-b). The geometry shows clear influence on metallicness (Fig. 9-c). It is believed that the perception of metallicness is related to the coverage of highlight (specular sheen), which can be enlarged by reducing the local surface curvature $[26,41]$. Our results match with this theory closely: an decreasing local surface curvature $\left(G_{\text {median }}\right)$ results in increasing metallicness in Fig. 9-c.

\subsubsection{Contrast of reflections}

As shown in Fig. 10-a, the rating of metal under illumination $I$ is significantly higher when compared to other material categories in terms of reflection contrast. This phenomenon can easily be observed in Fig. 4. Obviously, under illumination $I$, the highlight coverage on metal is much larger than for other material categories. Comparing with other environment maps, $I$ has the largest dynamic range (the highest $I_{\text {range }}$ and $I_{\text {std }}$ in Fig. 7) and is dominated by only one strongest point light (the smallest $I_{\text {area }}$ ), which results in wide and high contrast highlights on metal but narrow and lower-contrast highlight on other materials (refer to ghost under illumination $I$ in Fig. 4). This effect is amplified due to the different profiles of respective BRDFs shown in Fig. 5.

Another interesting observation holds for the blob. Except for illumination $I$, the blob gets similar rates on plastic, ceramic, and metal. Actually, this phenomenon is pronounced on all gloss-related attributes (Figs. 8 and 12). We show the rendered result for four geometries using the averaged BRDFs for these three material categories under illumination $E$ in Fig. 11 (similar for illumination $A$ and
$G)$. As discussed in Sect. 4.3.1, the gently changing smooth surface of blob enlarges the coverage of highlight and consequently boosts the perceived glossiness and all the other gloss-related attributes.

For the interaction between material category and illumination shown in Fig. 10-b, there are no significant differences observed between the combinations: fabric-A, plastic- $A$, and ceramic- $A$. This means the highly diffuse illumination like $A$ minimizes the differences between material categories [23].

\subsubsection{Sharpness of reflections}

Similar observations as for contrast of reflection under illumination $I$ also hold for sharpness of reflection in Fig. 12-a. The strong directional light $I$ consistently results in significantly higher rating for metal than for other material categories, where as can be seen in Fig. 5 the BRDF magnitude is larger for small $\theta_{h}$ and at the same time it is smaller for larger $\theta_{h}$. This contributes to stronger contrast between highlight and its surround for metals. As mentioned before, the highlight areas for metal tend to be overexposed due to the high reflectance property of metal, and the limit of current display system and tone mapping algorithm make this problem even more pronounced. Thus, one should avoid the highlight areas on metal when make the judgment on sharpness of reflection, which was explained by Serrano et al. [39] to experiment participants during the rating collection.

Except for $I$, the sharpness of reflection of dragon under other illuminations shows unintelligible patterns on different material categories (Fig. 12-a, c). It seems the tessellated-like bumpy surfaces on dragon greatly affect people's perception judgment on this attribute. We believe this is consistent with Marlow et al. [26] in their Experiment 2. 
Fig. 9 Estimated Marginal Means effect of metallicness for the interaction between $\mathbf{a}$. geometry, illumination, and material category (the height of each colored bar represents the value); b. material category and illumination; c. material category and geometry
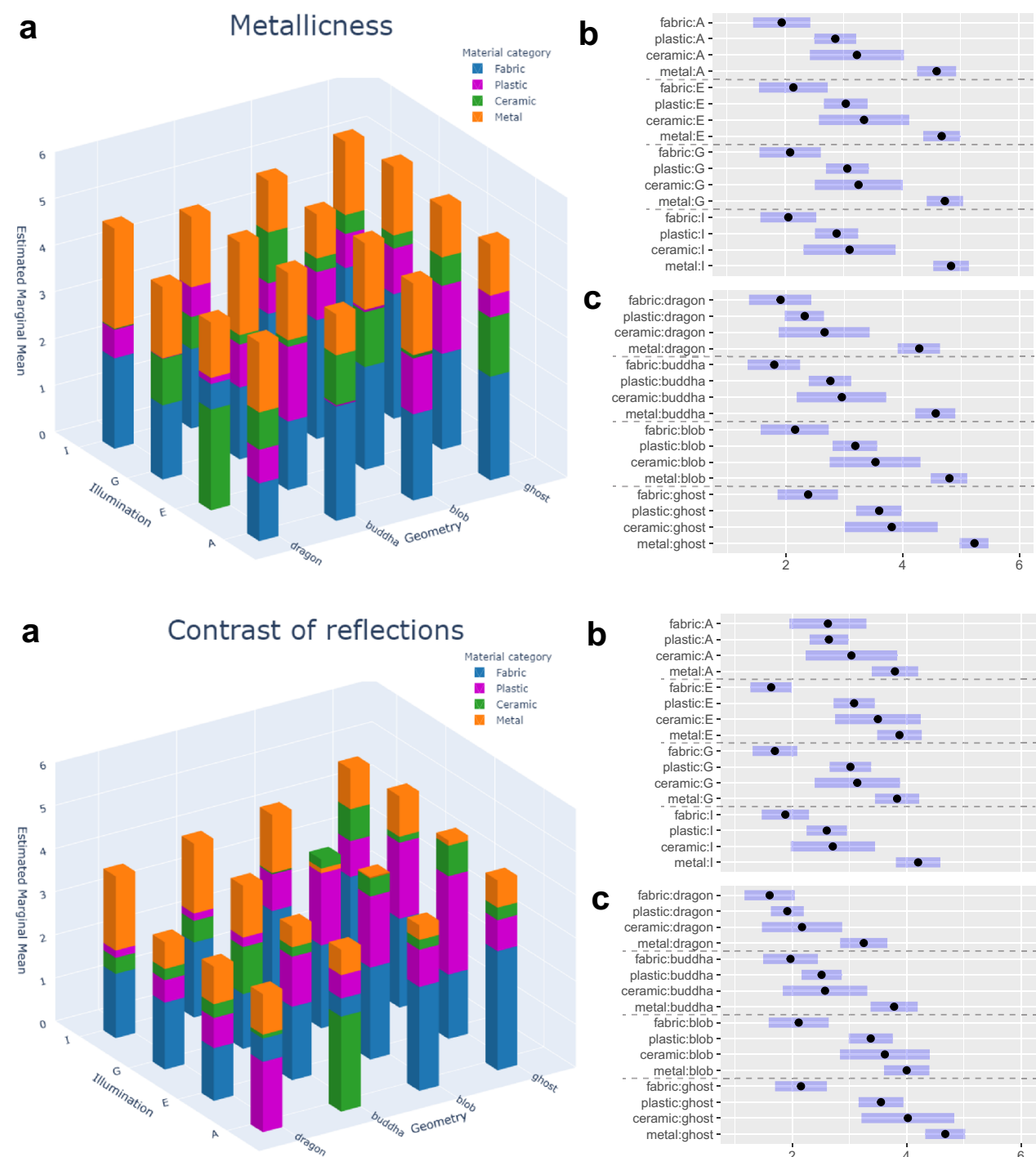

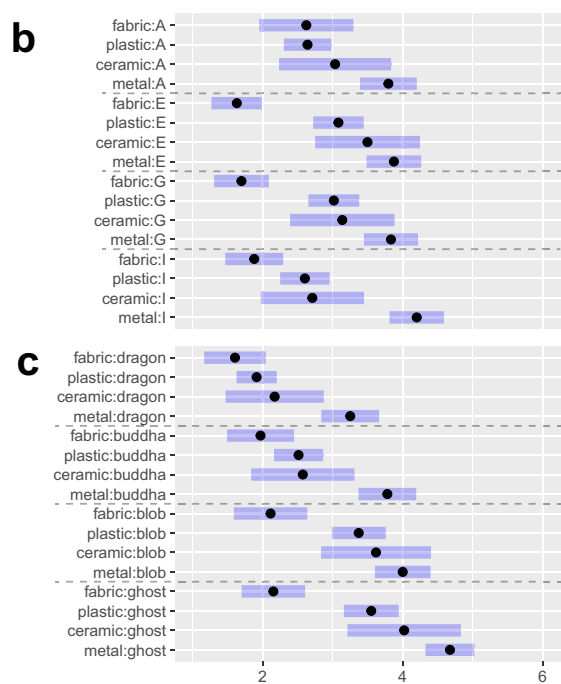

Fig. 10 Estimated Marginal Means effect of contrast of reflection for the interaction between a. geometry, illumination and material category (the height of each colored bar represents the value); b. material category and illumination; c. material category and geometry

\section{Conclusion}

In this work, we have investigated the joint effect of geometry, illumination, and material category on perceived material properties. In particular, we focus on metallicness and three gloss-related (glossiness, contrast of reflections, and sharpness of reflections) appearance attributes. First, we have found that the correlations between the appearance attributes for wood, paper, fabrics, leather, and natural materials are weak, and the attribute strength is significantly lower than for plastic, ceramic, and metal. The latter three material categories show strong correlations between different attributes, but also striking nonlinear relations between glossiness and contrast of reflections as well as sharpness of reflections can be observed. In general, materials should feature a certain glossiness before the contrast and sharpness of reflections start to be perceived. An interaction analysis between material categories and specific illuminations and geometries shows that metallicness is stronger for metals in all tested conditions; however, this is not the case for glossiness. For smooth, low curvature geometries such as blob and under smooth, low-frequency illumination $(A, E$, and $G)$, the differences in glossiness strength between metal, ceramic, and plastic are strongly reduced. This observation also holds for the contrast and sharpness of reflections attributes. On the other hand, for strong and directional illumination (such as I), the glossiness attribute is stronger for metals than for plastics or ceramics, regardless of geometry. The same effect can be observed for highly detailed and tessellated geometry (such as dragon), regardless of illumination. This observation also holds for the contrast of reflection attribute, but this is not the case for the sharpness of reflection attribute, where the dragon geometry makes the judgment of sharpness more difficult. In general, high-frequency features either in illumination or geometry increase the strength of gloss-related attributes for metal with respect to plastic and ceramic, but 


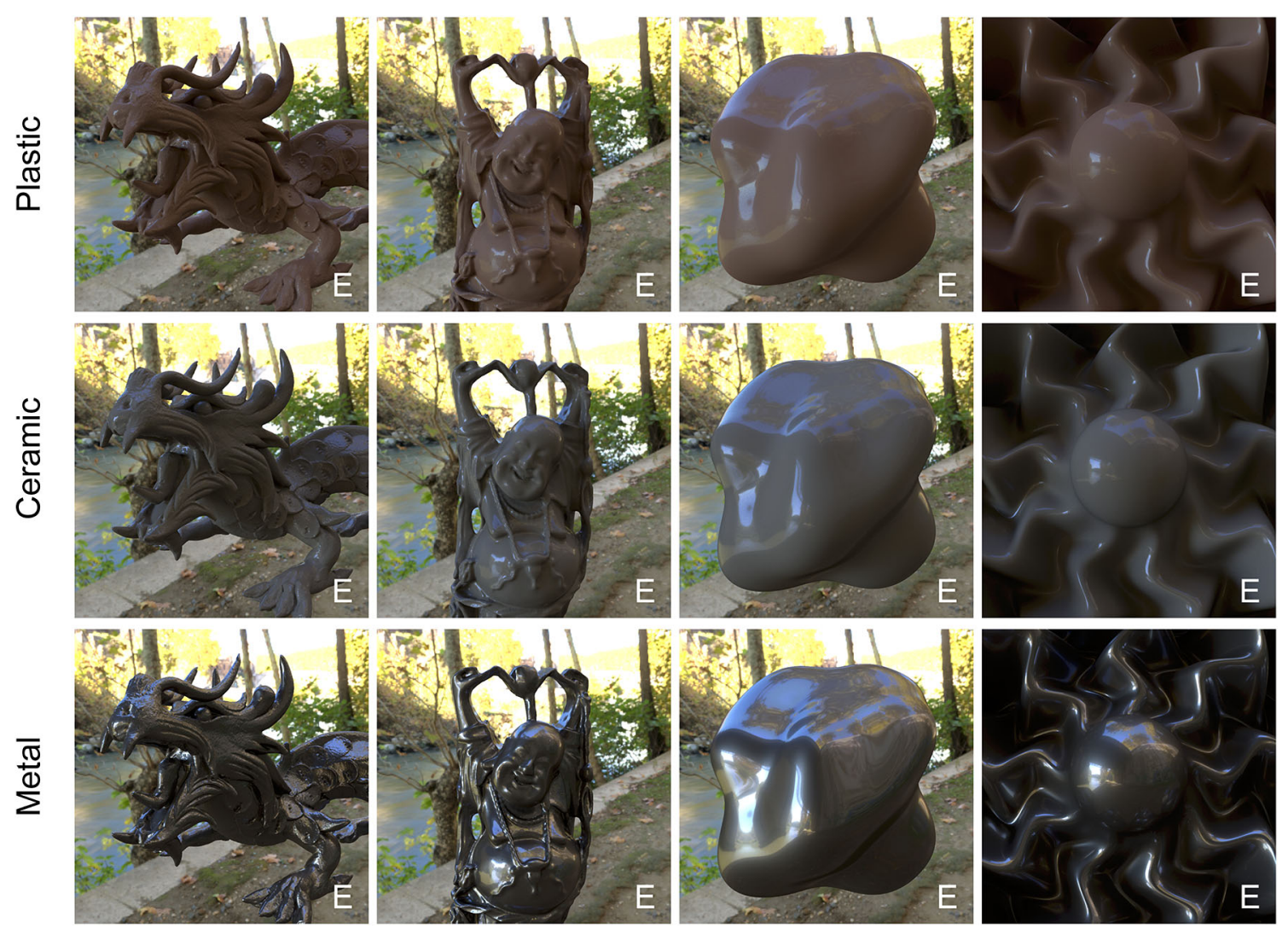

Fig. 11 Rendered images using three averaged BRDFs on four geometries under illumination $E$

Fig. 12 Estimated Marginal Means effect of sharpness of reflection for the interaction between a. geometry,

illumination, and material category; b. material category and illumination; c. material category and geometry

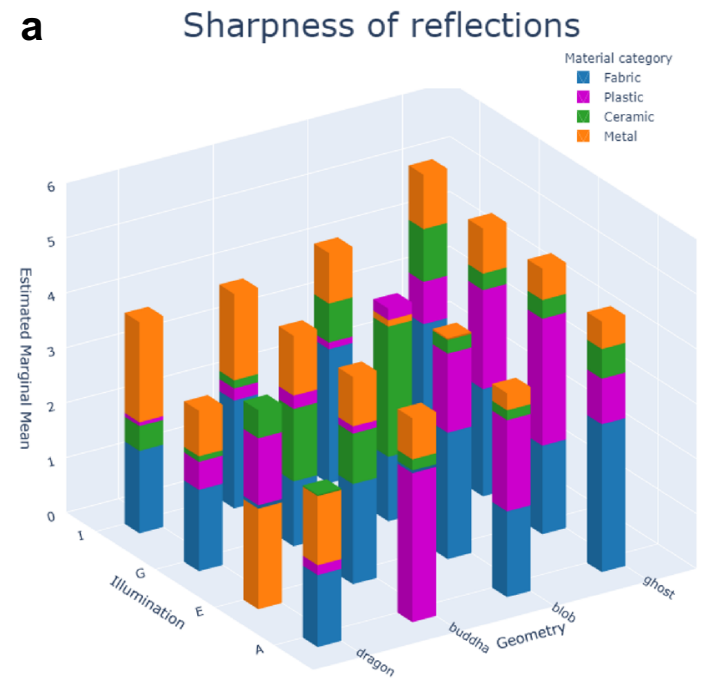

directional illumination seems to be a stronger factor here. The judgment of contrast and sharpness of reflections for less smooth geometries such as dragon or buddha is more difficult and shows higher variations for non-metal materials, including fabric. Again, this is not the case for strong directional illumination $(I)$.

An important characteristic of our work is that our findings are mostly related to displayed images, where due to dis- play luminance limitations as well as tone mapping, image contrast might be suppressed or even reflection details in highlight regions might be saturated (clamped). Such displayed image characteristics are conveyed in our data, and they are highly relevant for many visual computing tasks that involve human observers. However, our data for specific materials may differ from the perception of their real-world counterparts. Still, we believe that the general trends in our 
data remain valid, and we leave it as future work to investigate specific differences with the real-world conditions. The development of mixed reality displays would greatly facilitate this task.

We believe that our insights about the interactions between material category, illumination, and geometry contribute to expanding the knowledge about material perception and can be a useful guideline for design applications, in particular where designed objects are exposed under certain illuminations.

Acknowledgements This project has received funding from the European Union's Horizon 2020 research and innovation programme under the Marie Sklodowska-Curie, grant agreement $N^{\circ} 765911$ (RealVision) and from the European Research Council (ERC), grant agreement $\mathrm{N}^{\circ}$ 804226 (PERDY).

Funding Open Access funding enabled and organized by Projekt DEAL.

\section{Declarations}

Conflicts of interest The authors declare they have no conflict of interest.

Open Access This article is licensed under a Creative Commons Attribution 4.0 International License, which permits use, sharing, adaptation, distribution and reproduction in any medium or format, as long as you give appropriate credit to the original author(s) and the source, provide a link to the Creative Commons licence, and indicate if changes were made. The images or other third party material in this article are included in the article's Creative Commons licence, unless indicated otherwise in a credit line to the material. If material is not included in the article's Creative Commons licence and your intended use is not permitted by statutory regulation or exceeds the permitted use, you will need to obtain permission directly from the copyright holder. To view a copy of this licence, visit http://creativecomm ons.org/licenses/by/4.0/.

\section{References}

1. Adams, W.J., Kucukoglu, G., Landy, M.S., Mantiuk, R.K.: Naturally glossy: gloss perception, illumination statistics, and tone mapping. J. Vis. 18(13), 4-4 (2018)

2. Anderson, B.L.: Visual perception of materials and surfaces. Curr. Biol. 21(24), R978-R983 (2011)

3. Beck, J., Prazdny, S.: Highlights and the perception of glossiness. Percept. Psychophys. 30(4), 407-410 (1981)

4. Burley, B.: Physically-based shading at disney. In: ACM SIGGRAPH 2012 Courses, pp. 1-7 (2012)

5. Chadwick, A.C., Kentridge, R.: The perception of gloss: a review. Vision. Res. 109, 221-235 (2015)

6. Dror, R.O., Willsky, A.S., Adelson, E.H.: Statistical characterization of real-world illumination. J. Vis. 4, 821-837 (2004)

7. Dupuy, J., Jakob, W.: An adaptive parameterization for efficient material acquisition and rendering. ACM Trans. Graph. 37(6), 114 (2018)

8. Filip, J., Vávra, R.: Template-based sampling of anisotropic BRDFs. Comput. Graphics Forum 33(7), 91-99 (2014)
9. Fleming, R.W.: Visual perception of materials and their properties. Vis. Res. 94, 62-75 (2014)

10. Fleming, R.W.: Material perception. Ann. Rev. Vis. Sci. 3, 365-388 (2017)

11. Fleming, R.W., Bülthoff, H.H.: Low-level image cues in the perception of translucent materials. ACM Trans. Appl. Percept. 2(3), 346-382 (2005)

12. Fleming, R.W., Dror, R.O., Adelson, E.H.: Real-world illumination and the perception of surface reflectance properties. J. Vis. 3(5), 3-3 (2003)

13. Fleming, R.W., Nishida, S., Gegenfurtner, K.R.: Perception of material properties. Vis. Res. 115, 157-62 (2015)

14. Gkioulekas, I., Walter, B., Adelson, E.H., Bala, K., Zickler, T.: On the appearance of translucent edges. Comput. Vis. Pattern Recognit. 5528-5536 (2015)

15. Guennebaud, G., Gross, M.: Algebraic point set surfaces. In: ACM SIGGRAPH. 2007, pp. 23-es

16. Havran, V., Filip, J., Myszkowski, K.: Perceptually motivated BRDF comparison using single image. Comput. Graphics Forum 35, 1-12 (2016)

17. Hu, B., Guo, J., Chen, Y., Li, M., Guo, Y.: Deepbrdf: a deep representation for manipulating measured BRDF. Comput. Graphics Forum 39, 157-166 (2020)

18. Hunter, R.: Methods of determining gloss. J. Res. Natl. Bureau Standards 18, 19-39 (1937)

19. Hunter, R., Harold, R.: The Measurement of Appearance, 2nd edn. Wiley, New York (1987)

20. Kim, J., Marlow, P., Anderson, B.L.: The perception of gloss depends on highlight congruence with surface shading. J. Vis. 11(9), 4-4 (2011)

21. Kim, J., Marlow, P.J., Anderson, B.L.: The dark side of gloss. Nat. Neurosci. 15(11), 1590-1595 (2012)

22. Kim, J., Tan, K., Chowdhury, N.S.: Image statistics and the fine lines of material perception. i-Perception 7, 4 (2016)

23. Lagunas, M., Serrano, A., Gutierrez, D., Masia, B.: The joint role of geometry and illumination on material recognition. J. Vis. 21, 2 (2021)

24. Landy, Y.-X., Landy, M., Maloney, L.: Conjoint measurement of gloss and surface texture. Psychol. Sci. 19(2), 196-204 (2008)

25. Marlow, P., Kim, J., Anderson, B.L.: The role of brightness and orientation congruence in the perception of surface gloss. J. Vis. 11(9), 16-16 (2011)

26. Marlow, P.J., Anderson, B.L.: Generative constraints on image cues for perceived gloss. J. Vis. 13(14), 2-2 (2013)

27. Marlow, P.J., Anderson, B.L.: Material properties derived from three-dimensional shape representations. Vis. Res. 115, 199-208 (2015)

28. Marlow, P.J., Kim, J., Anderson, B.L.: The perception and misperception of specular surface reflectance. Curr. Biol. 22(20), 1909-1913 (2012)

29. Marlow, P.J., Todorović, D., Anderson, B.L.: Coupled computations of three-dimensional shape and material. Curr. Biol. 25(6), R221-R222 (2015)

30. Matusik, W., Pfister, H., Brand, M., McMillan, L.: A data-driven reflectance model. ACM Trans. Graph. 22(3), 759-769 (2003)

31. Motoyoshi, I., Matoba, H.: Variability in constancy of the perceived surface reflectance across different illumination statistics. Vis. Res. 53(1), 30-39 (2012)

32. Motoyoshi, I., Nishida, S., Sharan, L., Adelson, E.H.: Image statistics and the perception of surface qualities. Nature 447(7141), 206-209 (2007)

33. Nunnally, J.C.: Psychometric Theory 3E. Tata McGraw-hill Education (1994)

34. Olkkonen, M., Brainard, D.H.: Perceived glossiness and lightness under real-world illumination. J. Vis. 10(9), 5-5 (2010) 
35. Pellacini, F., Ferwerda, J.A., Greenberg, D.P.: Toward a psychophysically-based light reflection model for image synthesis. In: Proc. ACM SIGGRAPH, pp. 55-64 (2000)

36. Pont, S.C., te Pas, S.F.: Material-illumination ambiguities and the perception of solid objects. Perception 35(10), 1331-1350 (2006)

37. Rusinkiewicz, S.M.: A new change of variables for efficient BRDF representation. EGSR 11-22 (1998)

38. Schmid, A.C., Barla, P., Doerschner, K.: Material category determined by specular reflection structure mediates the processing of image features for perceived gloss. bioRxiv (2020)

39. Serrano, A., Chen, B., Wang, C., Piovarci, M., Seidel, H.-P., Didyk, P., Myszkowski, K.: The effect of shape and illumination on material perception: model and applications. ACM Trans. Graph. (2021)

40. Serrano, A., Gutierrez, D., Myszkowski, K., Seidel, H.-P., Masia, B.: An intuitive control space for material appearance. ACM Trans. Graph. 35, 6 (2016)

41. Todd, J., Norman, J.: The visual perception of metal. J. Vis. 18(3), 1-17 (2018)

42. Toscani, M., Guarnera, D., Guarnera, G.C., Hardeberg, J.Y., Gegenfurtner, K.R.: Three perceptual dimensions for specular and diffuse reflection. ACM Trans. Appl. Percept. 17(2), 1-26 (2020)

43. Vangorp, P., Laurijssen, J., Dutré, P.: The influence of shape on the perception of material reflectance. In: Proc. ACM SIGGRAPH, pp. 77:1-77:9 (2007)

44. Wills, J., Agarwal, S., Kriegman, D., Belongie, S.: Toward a perceptual space for gloss. ACM Trans. Graph. 28(4), 1-15 (2009)

45. Zhang, F., de Ridder, H., Barla, P., Pont, S.: A systematic approach to testing and predicting light-material interactions. J. Vis. 19(4), $1-22(2020)$

Publisher's Note Springer Nature remains neutral with regard to jurisdictional claims in published maps and institutional affiliations.

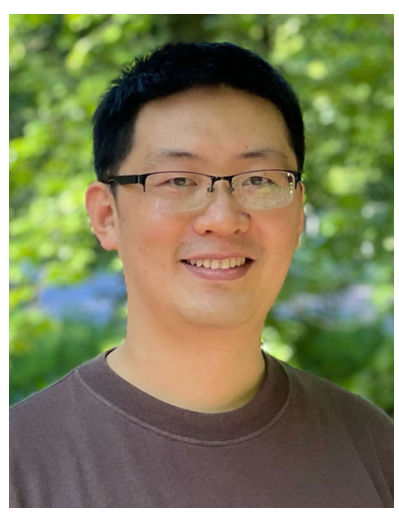

Bin Chen is a postdoctoral researcher in the computer graphics department of Max-PlanckInstitut für Informatik. He obtained his doctoral degree from City University of Hong Kong in 2020. His research interests include computational display, computational photography, deep learning, and perception. His work has been published in scientific journals such as Optics Express (OE), Jour nal of Laboratory Automation and ACM Transactions on Multimedia Computing, Communications, and Applications (TOMM) and leading international conferences proceedings like International Conference on Robotics and Automation (ICRA), SIGGRAPH.

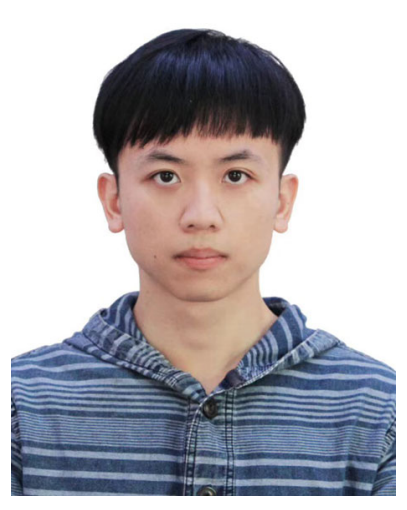

Chao Wang received the B.S. degree in information engineering from the University of Electronic and Science of China in 2017 and M.S. degree in computer engineering from Peking University in 2020 . He is currently pursuing $\mathrm{PhD}$ degree with the department of computer graphics in Max-Planck-Institut für Informa tik. His research interests include image processing, computer vision, material appearance, and deep learning.

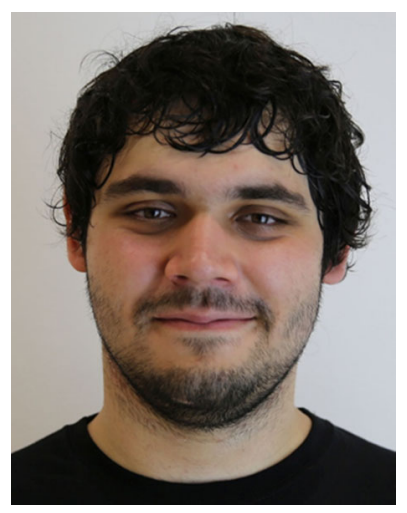

Michal Piovarči is a postdoctoral researcher at IST Austria. $\mathrm{He}$ received his doctoral degree at Università della Svizzera Italiana under the supervision of prof. Piotr Didyk. In his dissertation, Michal proposed to include hum an perception in the computational design and realization of objects' haptics and appearance. He demon strated that considering perception leads to improvements in computational fabrication, numerical simulation, and the creation of subjectively higher-quality prints. His pioneering work on perception-aware fabrication was awarded with one of the Eurographics $2021 \mathrm{PhD}$ awards.

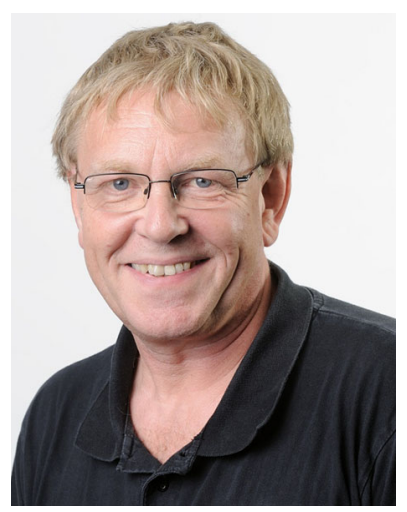

Hans-Peter Seidel is the scientific director and chair of the computer graphics group, MaxPlanck-Institut für Informatik and a professor of computer science with the Saarland University, Saarbrücken, Germany. He has been on the program committee of all major international graphics conferences, and chaired several of these events. His publication list includes more than 75 papers in ACM SIGGRAPH/the ACM Transactions on Graphics and more than 100 papers in Euro graphics/ CGF. He has received numerous awards for his work, including the DFG Leibniz Prize (2003) and the Eurographics Distinguished Career Award (2012). 


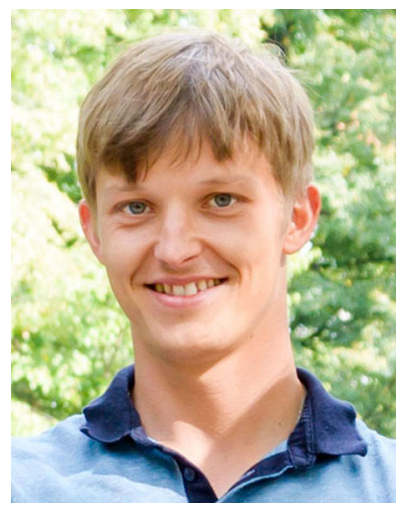

Piotr Didyk is a senior assistant professor at Università della Svizzera italiana (USI) in Switzerland. His research interests include human perception, new display technologies, image/ video processing, 3D printing, and computational fabrication. Before his current position, he was an Independent Research Group Leader at Saarland University, MMCI and Max Planck Institute for Informatics in Germany. He obtained his PhD from Saarland University and Max Planck Institute under Prof. Karol Myszkowski. In 2018, he was awarded an ERC Starting Grant, and in 2019, he was elected Junior Fellow of the European Association for Computer Graphics.

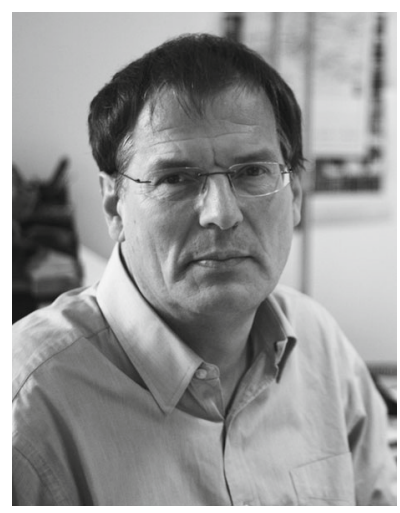

Karol Myszkowski is a senior researcher at the MaxPlanck Institut für Informatik, Saarbruecken, Germany. In the period from 1993 till 2000, he served as an associate professor in the Department of Computer Software at the University of Aizu, Japan. In the period from 1986 till 1992, he worked at Szczecin Technical University as well as at Integra, Inc. a company specialized in developing rendering and global illumination software. He received his $\mathrm{PhD}$ (1991) and habilitation (2001) degrees in computer science from Warsaw University of Technology (Poland). His research interests include global illumination and rendering, perception issues in graphics, high dynamic range imaging, and stereo $3 \mathrm{D}$.

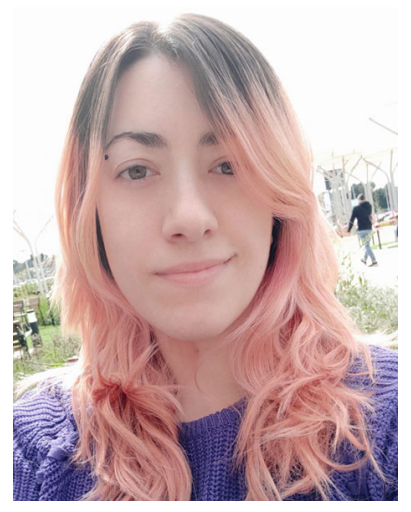

Ana Serrano is an assistant professor at the Centro Universitario de la Defensa (Zaragoza). Previously, she was a postdoctoral researcher at the Max-PlanckInstitut für Informatik. She received an Adobe Research Fellowship in 2017 and a NVIDIA Graduate Fellowship in 2018. Her thesis has been awarded with one of the Eurographics $2020 \mathrm{PhD}$ awards. Her research spans several areas of visual computing, in particular computational imaging, material appearance perception and editing, and virtual reality, with a focus on applying perceptually-motivated solutions. Her work has been published in top venues, including ACM Transactions on Graphics, Scientific Reports, and IEEE TVCG. 